\title{
ADAPTIVE RELEASE LEARNING PATHS TO MOTIVATE ACTIVE LEARNING AND ENGAGEMENT IN STUDENTS
}

\author{
Pavani Vemuri, Monique Snoeck and Stephan Poelmans \\ KU Leuven \\ 3000 Leuven, Belgium
}

\begin{abstract}
Learning Analytics (LA), a decade old emerging filed, has the potential to make data-informed decisions to improve the quality of Higher Education (HE). It can be a good tool for HE institutions to tackle problems like student retention and promote student success rates. While LA could involve studying the impact of socioeconomic variables such as age, work, gender, stage, status, etc., on student success; these variables cannot be addressed by a teacher. Study attitude on the other hand, may be affected by instructional design, study counselling and guidance with theory informed teaching interventions. Grounding first year bachelor's students in the culture of active learning in their first year itself, will help develop self-regulation strategies which will thereby improve success and retention for not just the first year but also to complete the bachelor program in the stipulated period. In this study, we analyze data sourced from across all the first-year bachelor's courses of an Economics and Business Faculty. The students are classified into different groups according to their summative scores and their LMS interaction behaviors are studied. in future work, the collection of data across different campuses, courses and student programs allows for a comparative analysis across different dimensions, thus allowing for the investigation of the generalizability of results by means of out-of-sample testing or models built on a single course's data. Additionally, the collection of data across three successive academic years will also allow for the out-of-time validation of findings, including the analysis of the impact of the COVID-19 pandemic on the students' behavior.
\end{abstract}

\section{KEYWORDS}

Technology Enhanced Learning, Learning Analytics, Automatic Feedback, Formative testing, Student retention, Student Success

\section{INTRODUCTION}

The use of learning management systems (LMS) and other learning technologies in higher education (HE) to improve the quality of education has become common place over past few years. The use of these technologies generates a lot of log data when students access these systems. Learning Analytics (LA) is an emerging field (still nascent, only a decade old) that grew due to the digitalization of education and the maturity of data mining technology (Ferguson, 2012). The HE sector looks to LA for solutions to issues like a student's learning progress, retention, satisfaction; improving teaching quality and innovations which result in an elevated learning experiences there by contributing towards institutional performance and ranking. Nevertheless, it important to know what the immediate goals of LA are, to choose the appropriate methods to be used. In search for factors that are predictive for learner success, our study focuses on factors that can be addressed by a teacher. While studying the impact of socioeconomic variables such as "Age, Work, Gender, Stage, Status" on student success is interesting (Hamoud, Hashim, \& Awadh 2018, p. 30), these variables cannot be addressed by a teacher. Study attitude on the other hand, may be affected by instructional design, study counselling and guidance.

Drop-out rates and success rates for first year students are a concern for many HE institutions. The Faculty of Economics and Business (FEB) at KU Leuven is no different as it experiences low success rates with its first-year students (less than half of the student's succeed only after a retake of the failed exams). The problem is not unique to FEB but prevails across all educational programs at KU Leuven (and even in Flanders). There has been a university-wide call for initiatives to find feasible solutions to this problem. The "Adaptive Learning Paths for ACtivation and Assessment of Students" (ALPACAS) project was initiated at FEB in 2019, with the central idea that - if more attention is given to first year students to ground them in the culture of active learning 
in their debut itself, they will develop self-regulation strategies early on; thereby improve success and retention for not just the first year but also to complete the bachelor program in the stipulated period. Among the key elements to achieve the goals of this project were: to utilize coordinated, uniform communication across all courses in a program; help didactic teams use formative assessments and adaptive learning paths including follow-up and feedback during the semester; and use LA to better inform teachers about their students.

One of the challenges for an institution/faculty-wide LA initiative is that courses even across one faculty may have a variety of teaching approaches as they are usually designed autonomously by their individual instructors or didactic teams; and hence diverse learning strategies maybe required. This diversity also presents an opportunity as different instructional designs can be used to investigate the generalizability of factors found to correlate with student success. In the context of the ALPACAS project, data was collected from all first-year courses across the entire faculty. This allows for the use of LA to investigate student behavior along different dimensions: comparing a same group of students' behaviors across different courses, comparing behavior in a same course across students from different programs or campuses, etc. LA is an appropriate tool in such context, to reflect on student behaviors to predict future behaviors and outcomes of students (Leitner, Khalil \& Ebner, 2017). Potential problems can be recognized in an early stage and appropriate teaching interventions can be used to mitigate issues like retention (Palmer, 2013) and promote student success. However, before engaging in predictive analytics and model building, it makes sense to start with an exploratory analysis to verify the assumption that active learning behaviors are associated to better learning outcomes.

The following research questions were tackled by the research presented in this paper:

RQ1: Does the exploratory analysis of the data confirm that different behavior can be associated with different levels of student success?

RQ2: Does exploratory analysis allow to distinguish between different factors correlating with learner success?

The remainder of the paper is organized as follows: Section 2 presents the data collected in the context of the ALPACAS project. Section 3 presents some results of the exploratory analysis. Section 4 discusses avenues for further research and concludes the paper.

\section{DATA DESCRIPTION}

Each year, approximately 4000+ students enroll into (one of) the 10 programs (taught in English/Dutch languages) offered by FEB at its 4 locations. Each program has 4-5 courses taught each semester summing up to 9-11 courses per year. An instructor or didactic team teaching the course has autonomy over how the course is taught, the pedagogy and use of tools etc. There are 110+ courses taught in the first-year bachelor programs excluding languages. The primary datasets were extracted from the foundation technologies (Gasevic et al., 2019) of an HE institution: the Toledo-Blackboard LMS used at KU Leuven; and the student information systems (SIS). Due to strict GDPR guidelines in Europe, the socio-demographic data was not collected from the SIS but only student enrollment data and their summative scores were sourced. As the datasets are across multiple systems, it was mapped appropriately and pseudonymized in accordance with the university guidelines on data management. Secondary datasets are yet to be sourced from other sources like the Kaltura platform (a video cloud platform that integrates with an LMS) and we are currently also working on collecting longitudinal data across three academic years in the next data extracts. The first extract of the data was transversal in nature collected over the academic year 2019-2020. There are numerous dimensions to consider when analyzing this data. A course can be taught by different teachers on different campuses, in different academic years, or in different semesters. Data was simplified using a hierarchical structure - students in courses taught by teachers in each semester belonging to programs offered in campuses. The Toledo extension of the LMS allows to collect more than just trace data (navigation between webpages). The interaction of students with the LMS is captured in specific clicks termed as Events.

\section{PRELIMINARY ANALYSIS}

As a part of the preliminary analysis, the student behaviors across the semester were visualized in the courses. First, the content types used in courses were identified. Each course used various content types per the didactic 
team's choice of the course design. To compare events between courses, and to know the scale of how instructors used the LMS functionalities, (a) the number of learning items per content type and (b) number of events per content type were examined for each course. Next, students were categorized according to their summative scores into four categories (Student_class) of students: "Excellent" (scores above 16/20), "Successful" (between 10 and 15/20), "Able to Push" (scores 7, 8 or 9/20), and "Poor" students (score below 7/20). Student behavior during the semester was captured as average events per week per Student_class.

Figure 1 shows student behavior in two courses Marketing and Global Economy offered in the business engineering program (HIR). Firstly, we can distinctly notice (also in figure 2) that Excellent students worked hard consistently throughout the semester (followed by Successful) than any other Student_class. We also noticed that this is the general trend across most courses. The Poor students work very hard mostly at the semester-end and have the highest number of exam-period events in some courses. These, among other observations of visualizations confirm RQ1 that behavior can be associated with different levels of student success.

Notice that the average events are higher for Global Economy with the intensive use of the flipped classroom approach with adaptive paths by offering 11 formative tests with feedback which were actively used (not shown here); compared to Marketing which offered no tests at all. While longitudinal studies may give more insights into courses and student behaviors, it is clear that not all courses are built alike and evidently further analysis is needed to understand what makes the success rates of one course higher than others.
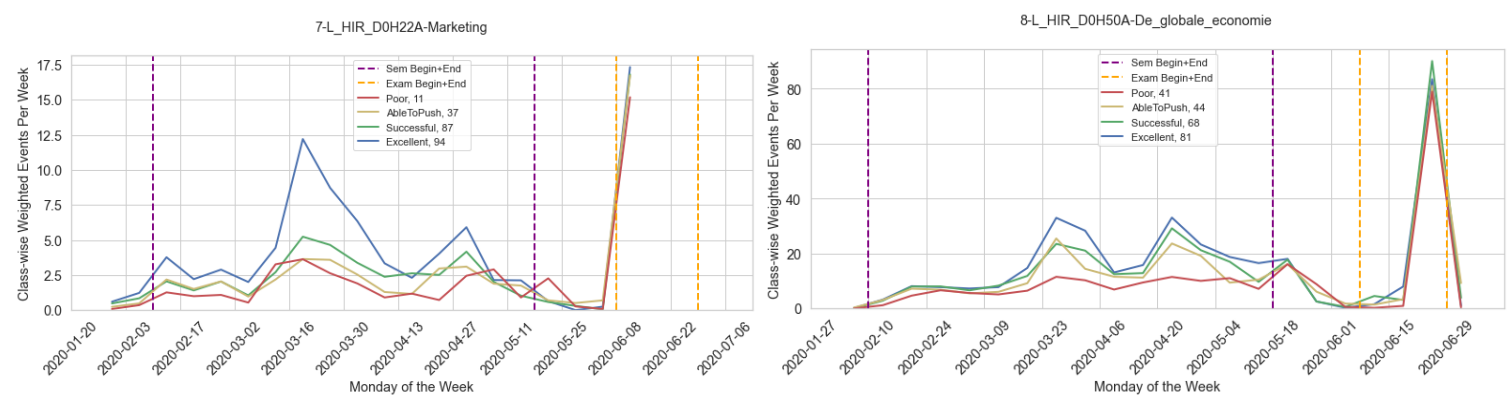

Figure 1. Average events per student group per week in the 'Marketing' (left) \& 'Global Economy' (right), HIR Program

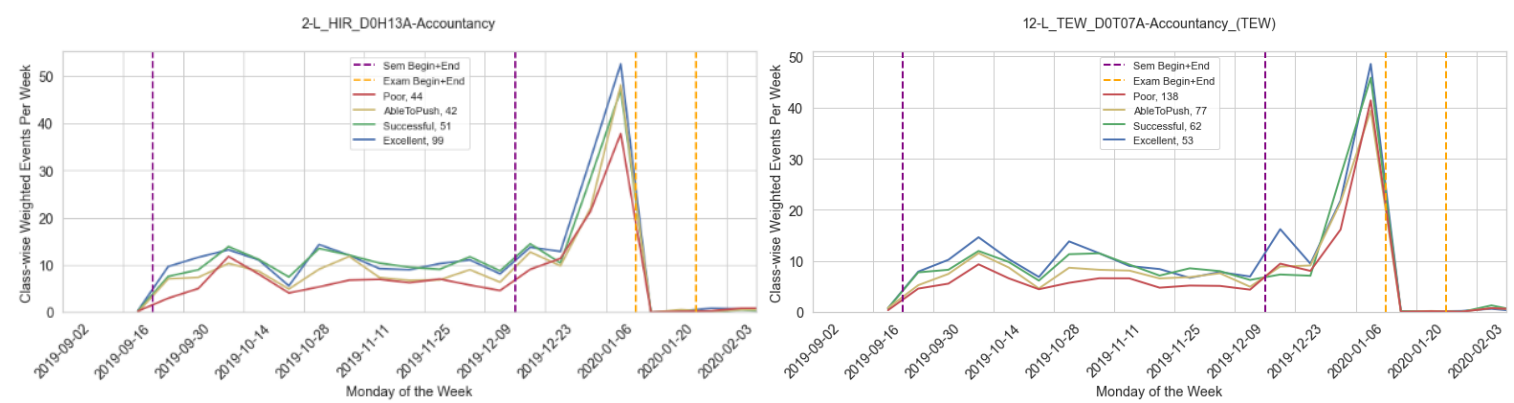

Figure 2. Average events per student group per week: in the 'Accountancy' course, (left) HIR and (right) TEW program

Secondly, consider the Figure 2 which shows student behavior in the Accountancy course followed by two distinct cohorts. The management students (TEW) have a lesser stronger quantitative profile than those of the business engineering (HIR). The numbers in the legend of each graph indicate the number of students in that specific Student_class. Notice that in both cohorts, class-wise student behaviors are very much alike despite their demographic differences. Perhaps HIR students are more successful in this course due to their quantitative profiles and additional interventions might be required for TEW students. Quantitative profile of a student can be a factor that can predict student success in some courses. Additionally, in both figures $1 \& 2$, the peaks in activity before the exam periods can be ode to summative assignments, specific formative tests or mock exams. Excellent student_class (followed by Successful) have higher activity than others as indicated by these pre-exam period peaks. But it is not straight forward to infer from the preliminary analysis (RQ2) or distinguish which other factors affect and are correlated with success without further in-depth analysis. 


\section{CONCLUSION AND FUTURE WORK}

Collecting such a large amount of data, checking its relevance, and analyzing it in HE at a formal university across so many courses and programs is very uncommon. Gasevic et al. (2019) mention that predictive modeling and simulation or analytics informed intervention was not carried out on a large scale at institutions. Herodotou et al. (2019) report a large-scale implementation of predictive LA in HE but at the open university in UK with mostly completely online courses. In future work, our research team plans to conduct predictive analysis to identify at risk students in each course separately; compare how students distribute activity across courses in a semester compare the success of different cohorts of students attending the same courses, analyzing impact of COVID-19 pandemic on student behavior, etc. Especially in the second semester, with the data from first semester behavioral analysis and summative scores; and early prediction analysis with early second semester data; teaching interventions or student counselor interventions can be planned to support at risk students.

The initial exploration of the data demonstrates the data to be rich enough to analyze various aspects of student behavior. It can be inferred from exploratory analysis that different behavior can be associated with different levels of student success. While some factors like quantitative profiles are distinguishable from exploratory analysis as predictors of success, an in-depth analysis is needed for exploring many other factors of prediction. The collection of data across different campuses, courses and student programs allows for a comparative analysis across different dimensions, thus allowing for the investigation of the generalizability of results by means of out of sample testing or models build on a single course's data. Hence on one hand, analysis needs to be conducted multi-dimensionally at the course, semester, program and campus levels. On the other hand, equally important is to equip teachers with the know-hows of making informed teaching interventions. The ALPACAS project also aims to bring the instructors together to learn not just from LA but also each other's practices. As we aim towards capturing longitudinal datasets, the collection and analysis of data across three successive academic years will also allow for the out-of-time validation of findings, including the analysis of the impact of the COVID-19 pandemic.

\section{REFERENCES}

Ferguson, R. (2012). Learning analytics: drivers, developments and challenges. International Journal of Technology Enhanced Learning, 4(5/6), pp. 304-317.

Gasevic, D., Tsai, Y., Dawson, S., Pardo, A. (2019). How do we start? An approach to learning analytics adoption in higher education. The International Journal of Information and Learning Technology, 36(4), pp. 342-353.

Hamoud, A. K., Hashim, A. S., Awadh, W. A. (2018). Predicting Student Performance in Higher Education Institutions Using Decision Tree Analysis. Intl. Journal of Interactive Multimedia and Artificial Intelligence, 5(2), pp. $26-31$.

Herodotou, C., Rienties, B., Boroowa, A. et al. (2019). A large-scale implementation of predictive learning analytics in higher education: the teachers' role and perspective. Education Tech Research Dev, 67, pp. 1273-1306.

Jeroen J. G. M. and Paul A. K. (2017) Ten steps to complex learning: A systematic approach to four-component instructional design.3rd edn. New York: Routledge.

Leitner, Khalil and Ebner (2017). 'Learning analytics in higher education-a literature review' in A. Peña-Ayala (ed.). Learning analytics: fundaments, applications, and trends. Springer., pp. 1-24.

Palmer S. (2013). Modelling engineering student academic performance using academic analytics. Int J Eng Educ 29(1), pp. 132-138. 